Reprod. Nutr. Dévelop., 1986, 26 (2 B), 669-676.

\title{
Evolution de la capacité d'estérification des acides gras dans les adipocytes de rat avant le sevrage : effet de la suralimentation lactée
}

\author{
Isabelle DUGAIL, Francine DUPUY, Annie QUIGNARD-BOULANGÉ \\ INSERM U.177, Unité de Recherches sur la Physiopathologie de la Nutrition, \\ 15, rue de l'Ecole-de-Médecine, 75270 Paris Cedex 06.
}

Summary. Development of the fatty esterification pathway in rat fat cells during the suckling period. Effect of early overfeeding.

During the suckling period, lipid storage in rat adipose tissue arises from exogenous triglycerides, as milk contains a large amount of lipid. In the present work, we studied the effect of overfeeding during the suckling period on the developmental pattern of the fatty acid esterification pathway in fat cells.

Early overfeeding was induced by restricting litter size; at 2 days of age, the pups were randomly distributed to form litters of 4 (overfed group) or 8 (controls). During the suckling period the activity of lipoprotein lipase (LPL), glycerophosphate dehydrogenase (G3PDH), glycerophosphate acyl transferase (GPAT) and acyl CoA ligase were measured in isolated fat cells from the inguinal adipose tissue. As early as 10 days of age, plasma triglyceride levels were increased in overfed pups as compared to the controls and, in these animals, the adipose tissue was overdeveloped, mainly due to fat cell hypertrophy.

At that age, overfeeding induced a significant increase in LPL and GPAT activities, but acyl CoA ligase and GPDH were not modified in overfed pups. Fat cell hypertrophy appeared to be responsible for the increased GPAT activity in the overfed pups. On the contrary, the effect of overfeeding on LPL activity was not only related to fat cell enlargement, as the enzyme activity continued to increase in the overfed rats when expressed per unit of cell surface area.

These results show that overfeeding during the suckling period rapidly induced an increase in the fat storage capacity of the adipose tissue. This suggests that circulating triglyceride levels could regulate directly or through hormonal control the activity of the fatty acid esterification pathway in fat cells.

\section{Introduction.}

Le rat naît dépourvu de tissu adipeux blanc, mais ce tissu commence à se développer dès les premiers jours de la vie. Pendant toute la période d'alimentation lactée, le raton consomme un régime hyperlipidique et de ce fait l'essentiel des lipides stockés dans le tissu adipeux en cours de développement, provient des triglycérides d'origine exogène. Les lipoprotéines circulantes (chylomicrons-VLDL) sont hydrolysées par la lipoprotéine lipase (LPL) située dans la paroi des capillaires 
sanguins, et les acides gras libérés pénètrent dans les adipocytes où ils sont réestérifiés et stockés. Dans ces conditions, la quantité de triglycérides stockés dans un adipocyte doit dépendre de sa capacité d'estérification des acides gras et de la quantité de lipides circulants.

Le but de ce travail est d'étudier pendant la période qui précède le sevrage, l'évolution de l'activité de la LPL et des principaux enzymes de la voie d'estérification des acides gras dans le tissu adipeux, et son éventuelle régulation par le taux de triglycérides circulants. La suralimentation lactée d'un groupe d'animaux a été induite par réduction de la taille des portées (Kennedy, 1957 ; Widdowson et McCance, 1960).

\section{Matériel et méthodes.}

Tous les animaux utilisés dans cette étude (rats Wistar A.F.) ont été élevés dans notre animalerie. Les femelles allaitantes sont gardées en cage individuelle et ont librement accès à la nourriture (Biscuits UAR) et à l'eau de boisson. A l'âge de 2 jours, les ratons sont répartis au hasard pour former des portées de 4 ou 8 petits. A 2, 5, 10 et 15 jours, les animaux sont sacrifiés par décapitation et leur sang est recueilli. Le tissu adipeux inguinal des animaux d'une même portée est dissocié par la collagénase (Rothblatt et de Martinis, 1977) pendant $1 \mathrm{~h}$. Puis la suspension cellulaire est filtrée (filtre nylon mailles $190 \mu \mathrm{m}$ ) et après décantation des adipocytes, la phase inférieure est éliminée par aspiration. Les adipocytes sont lavés 2 fois avec une solution saline (Hanks) puis repris par un tampon approprié aux dosages envisagés. Le volume adipocytaire moyen est déterminé à l'aide d'un Coulter-Counter (ZBI-C1000 Channelyzer) équipé d'un tube à orifice de $200 \mu \mathrm{m}$ de diamètre. Le nombre d'adipocytes est calculé en divisant le poids de lipides du tissu par le poids moyen d'un adipocyte. Le contenu tissulaire en lipides est mesuré par gravimétrie après extraction par la méthode de Folch et al. (1957). Les activités de la glycérol-3-phosphate déshydrogénase (G3PDH), I'acyl-CoA ligase, et glycérol-3-phosphate acyl transférase (GPAT) ont été mesurées sur le surnageant obtenu par centrifugation de l'homogénat (15 min à $1500 \mathrm{~g}$ ). Le dosage de la G3PDH a été fait selon la technique de Kozak et Jensen (1974) modifiée par Wise et Green (1979), les activités GPAT et acyl CoA ligase selon les techniques de Jamdar et Fallon (1973) et Tanaka et al. (1979) respectivement. L'activité de la lipoprotéine lipase a été mesurée sur une poudre acétonique d'adipocytes remise en solution dans un tampon $\mathrm{NH}_{4} \mathrm{Cl}-\mathrm{NH}_{4} \mathrm{OH} 50 \mathrm{mM}, \mathrm{pH} 8,1$ contenant $4 \mathrm{UI} / \mathrm{ml}$ d'héparine, selon la technique décrite par Nilsson-Ehle et Schotz (1976). Les résultats sont exprimés en nmoles de produit formé par minute et par mg de protéines dosées dans l'homogénat (Lowry et al., 1951) ou par $10^{6}$ cellules (LPL).

\section{Résultats.}

Le tableau 1 montre l'évolution du poids corporel des 2 groupes d'animaux. Dès l'âge de 10 jours, les rats élevés par portées de 4 sont significativement plus lourds que leurs témoins, et cette différence augmente encore à 15 jours. Dès l'âge de 10 jours, une hypeitriglycéridémie et une hyperinsulinémie s'installent chez les animaux suralimentés et persistent jusqu'au sevrage (tabl. 1). 
Développement du tissu adipeux. - Le développement du tissu adipeux inguinal est plus rapide chez les rats élevés par portées de 4 que chez les témoins (tabl. 1) ; dès l'âge de 10 jours, la différence devient significative. Le tableau 2

TABLEAU 1

Effet de la suralimentation lactée sur le poids corporel, le poids du tissu adipeux inguinal, la triglycéridémie et l'insulinémie.

\begin{tabular}{|c|c|c|c|c|c|}
\hline $\begin{array}{l}\text { Age } \\
\text { jours }\end{array}$ & $\begin{array}{l}\text { Taille } \\
\text { portée }\end{array}$ & $\begin{array}{l}\text { Poids } \\
\text { corporel } \\
\text { (g) }\end{array}$ & $\begin{array}{c}\text { Triglycéridémie } \\
(\mathrm{g} / \mathrm{l})\end{array}$ & $\begin{array}{c}\text { Insulinémie } \\
(\mu \mathrm{U} / \mathrm{ml})\end{array}$ & $\begin{array}{l}\text { Poids tissu } \\
\text { adipeux } \\
\text { inguinal } \\
\text { (mg) }\end{array}$ \\
\hline 2 & & $6,9_{(7)} \pm 0,1$ & $2,49 \pm 0,18$ & $75,52 \pm 20,38$ & $38 \frac{ \pm}{(7)} 5$ \\
\hline 5 & $\begin{array}{l}4 \\
8\end{array}$ & $\begin{array}{l}12,4 \underset{(7)}{ \pm} 0,6 \\
11,0 \underset{(7)}{ \pm} 0,4\end{array}$ & $\begin{array}{l}2,42 \pm 0,27 \\
2,74 \underset{(10)}{ \pm} 0,26\end{array}$ & $\begin{array}{l}52,70 \pm 11,69 \\
47,58 \frac{ \pm}{(8)} 9,29\end{array}$ & $\begin{array}{c}86 \pm 14 \\
73 \pm \frac{(7)}{(7)} 12\end{array}$ \\
\hline \multirow{2}{*}{10} & 4 & $\underset{\substack{(19) \\
* *}}{24,2 \pm 0,8}$ & $\begin{array}{c}3,06 \pm 0,37 \\
(11) \\
*\end{array}$ & $35,90 \underset{*}{(12)} \underset{*}{ \pm} 2,85$ & $\begin{array}{c}283 \pm 29 \\
(19) \\
* *\end{array}$ \\
\hline & 8 & $21,4 \pm 0,6$ & $2,15 \underset{(22)}{ \pm} 0,16$ & $28,30 \underset{(24)}{ \pm} 1,99$ & $188 \pm 14$ \\
\hline \multirow{2}{*}{15} & 4 & $\begin{array}{c}38,8 \pm 1,0 \\
(18) \\
* * *\end{array}$ & $\underset{\substack{(24) \\
* *}}{2,32} 0,16$ & $51,16 \underset{\substack{(22) \\
* * *}}{ \pm} 5,67$ & $\underset{\substack{(18) \\
* * *}}{629 \pm} 43$ \\
\hline & 8 & $\begin{array}{c}30,4 \pm 1,0 \\
(16)\end{array}$ & $1,90 \underset{(40)}{ \pm 0,07}$ & $27,24 \underset{(38)}{ \pm} 2,94$ & $\underset{(16)}{242 \pm 22}$ \\
\hline
\end{tabular}

Chaque valeur représente une moyenne \pm écart-type avec le nombre de portées étudiées entre parenthèses. Entre les 2 groupes, les valeurs sont comparées à l'aide du test $t$ de Student-Fischer, les niveaux de signification sont les suivants: ${ }^{*} P<0,05,{ }^{*} P<0,01$, ${ }^{* *} P<0,001$.

TABLEAU 2

Cellularité du tissu adipeux inguinal.

\begin{tabular}{|c|c|c|c|c|c|}
\hline & $\begin{array}{l}\text { Taille } \\
\text { portée }\end{array}$ & 2 jours & 5 jours & 10 jours & 15 jours \\
\hline \multirow{2}{*}{$\begin{array}{c}\text { Volume } \\
\text { d'un } \\
\text { adipocyte } \\
\left(\times 10^{3} \mu m^{3}\right)\end{array}$} & 4 & \multirow{2}{*}{$9,14 \underset{(7)}{ \pm} 1,03$} & $\begin{array}{c}30,4 \underset{(7)}{ \pm, 49} \\
\text { NS }\end{array}$ & $\underset{(19)}{62,8} \underset{* *}{ \pm} 5,76$ & $\underset{\substack{(18) \\
* * *}}{76,06}$ \\
\hline & 8 & & $20,6 \underset{(7)}{ \pm} 3,30$ & $44,6 \underset{(18)}{ \pm} 3,04$ & $43,6_{(16)}^{ \pm} 4,26$ \\
\hline \multirow{3}{*}{$\begin{array}{c}\text { Nombre } \\
\left.\text { d'adipocytes }^{\prime} \text { (106/2 pads }\right)\end{array}$} & 4 & \multirow{3}{*}{$1,15 \underset{(7)}{ \pm 0,33}$} & $1,05 \underset{(7)}{ \pm} 0,11$ & $\begin{array}{c}2,84 \pm 0,28 \\
(18)\end{array}$ & $5,69_{(17)}^{ \pm} 0,52$ \\
\hline & & & NS & NS & * \\
\hline & 8 & & $1,25 \underset{\text { (7) }}{ \pm} 0,23$ & $\begin{array}{c}2,57 \pm 0,30 \\
(17)\end{array}$ & ${ }_{(15)}^{3,32 \pm 0,40}$ \\
\hline
\end{tabular}

Les valeurs représentent des moyennes \pm leur écart-type avec le nombre de portées étudiées entre parenthèses. Les différences sont significatives au seuil : ${ }^{*} \mathbf{P}<0,05,{ }^{*}{ }^{*} \mathbf{P}<0,01$, *** $\mathrm{P}<0,001$ (test $\mathrm{t}$ de Student-Fischer). 
montre l'évolution de la taille et du nombre des adipocytes dans le tissu inguinal. Dans les 2 groupes, la taille des adipocytes augmente entre 2 et 10 jours : L'augmentation est plus rapide chez les animaux suralimentés et à 10 jours, le volume des adipocytes est plus élevé chez ces animaux que chez les témoins. Le nombre d'adipocytes augmente de manière identique dans les 2 groupes d'animaux pendant les 10 premiers jours. A 15 jours, par contre, les rats suralimentés présentent une hyperplasie adipocytaire puisque leur nombre d'adipocytes est 2 fois plus grand que celui de leurs témoins.

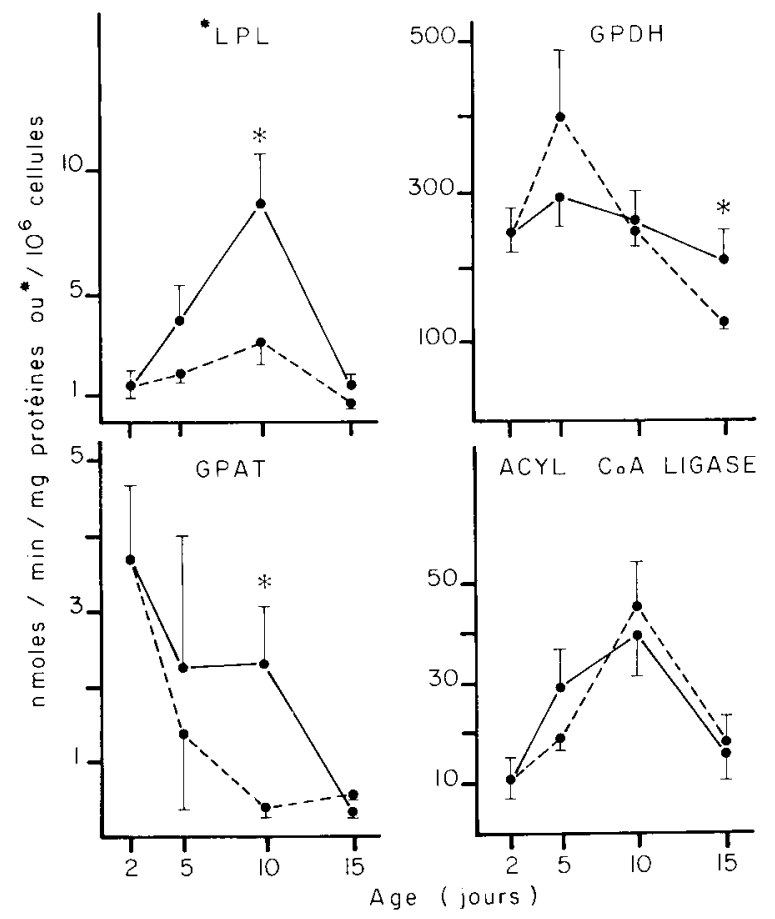

FIG. 1. - Effet de la suralimentation lactée sur les activités enzymatiques des adipocytes. Les rats témoins sont figurés en pointillé. Les rats suralimentés en traits pleins. Chaque point représente une valeur moyenne calculée sur au moins 4 portées, assortie de son écart-type. Les valeurs sont comparées à l'aide du test $t$ de Student-Fischer. Les niveaux de signification sont les suivants : $* P<0,05$.

Activités enzymatiques (fig. 1). - Pour les 2 groupes d'animaux, l'activité LPL par cellule augmente régulièrement mais de façon plus importante chez les rats suralimentés et à 10 jours, l'enzyme est 3 fois plus active que chez les témoins, cette différence reste significative lorsque l'activité est exprimée par unité de surface adipocytaire (tabl. 3). A 15 jours, l'activité LPL chute brutalement. L'activité de la GPDH dans les adipocytes, est stable pendant les 10 premiers jours, puis elle diminue de $50 \%$ chez les témoins, mais reste élevée chez les rats suralimentés : à 15 jours, ceux-ci présentent une activité GPDH significativement plus importante que leurs témoins. Le profil d'évolution de l'acyl CoA ligase dans les adi- 
pocytes présente également un pic d'activité à l'âge de 10 jours. Cependant, la suralimentation ne modifie pas l'activité de cette enzyme. L'activité de la GPAT diminue rapidement au cours du développement, mais cette chute est moins rapide chez les animaux suralimentés que chez les témoins : à 10 jours, l'enzyme est 3 fois plus active chez ces animaux que chez les témoins. Cette différence n'apparaît plus à 15 jours. L'effet de la suralimentation disparaît lorsque l'activité GPAT est exprimée par unité de surface adipocytaire (tabl. 3).

TABLEAU 3

Activités enzymatiques/unité de surface adipocytaire.

\begin{tabular}{|c|c|c|c|c|c|}
\hline \multicolumn{6}{|c|}{ Age (jours) } \\
\hline & $\begin{array}{c}\text { Nombre } \\
\text { de petits } \\
\text { par portée }\end{array}$ & 2 & 5 & 10 & 15 \\
\hline \multirow{3}{*}{$\begin{array}{c}\mathrm{LPL} \\
\mathrm{pmoles} / \mathrm{h} / \mathrm{mm}^{2}\end{array}$} & 4 & \multirow{3}{*}{$47 \underset{(7)}{ \pm} 17$} & $58 \pm 24$ & $84 \pm 22$ & $10 \frac{ \pm}{(7)}^{3}$ \\
\hline & & & & * & \\
\hline & 8 & & $35 \pm \frac{ \pm}{(7)} 10$ & $34 \underset{(10)}{ }{ }^{3} 10$ & $9 \underset{(7)}{ \pm} 2$ \\
\hline \multirow{3}{*}{$\begin{array}{c}\text { GPAT } \\
\text { pmoles } / \mathrm{min} / \mathrm{mm}^{2}\end{array}$} & 4 & & $0,8 \underset{(5)}{ \pm} 0,6$ & $0,5 \underset{(8)}{ \pm 0,2}$ & $0,05 \underset{(7)}{ \pm} 0,01$ \\
\hline & & $2,9 \underset{(4)}{ \pm} 0,8$ & & & \\
\hline & 8 & & $0,5 \underset{(5)}{ \pm} 0,4$ & $0,2 \pm 0,1$ & $0,14 \underset{(7)}{ \pm} 0,05$ \\
\hline
\end{tabular}

Les valeurs représentent des moyennes \pm leur écart-type avec le nombre de déterminations entre parenthèses. ${ }^{*}$ Différence significative entre les 2 groupes d'animaux au seuil : $P<0,05$ (test $t$ de Student-Fischer).

\section{Discussion.}

Le rôle de la LPL du tissu adipeux est particulièrement important lorsque l'alimentation est riche en lipides puisqu'elle contrôle l'entrée des acides gras dans les adipocytes. Nos résultats montrent que l'activité de cette enzyme augmente pendant la première moitié de la période d'alimentation lactée et diminue de façon importante à l'approche du sevrage. Le même profil (pic d'activité à l'âge de 10 jours) a été décrit par Cryer et Jones (1978) dans plusieurs autres localisations adipeuses, cela suggère que ce pic correspond à un processus ontogénique de développement, qui ne semble pas médié par l'insuline dont la concentration circulante décroît régulièrement pendant toute la période d'alimentation lactée. Dans les adipocytes du tissu sous-cutané la voie de synthèse des glycérolipides est plus active chez les animaux nouveaux-nés que chez les rats sevrés (Hahn et Greenberg, 1968). En accord avec cette observation, nos résultats montrent que l'acti- 
vité GPAT décroît régulièrement jusqu'au sevrage. Cependant au cours du développement, la synthèse varie considérablement en fonction de la localisation adipeuse (Jamdar et Osborne, 1981). Nos résultats concernant l'évolution de I'activité de la GPDH dans les adipocytes du tissu sous-cutané sont tout à fait comparables à ceux obtenus par Cook et Kozak (1982) chez la souris. Ainsi le profil de développement de cette activité enzymatique est indépendant de l'espèce ou de la souche utilisée. Cependant, ces auteurs ont rapporté d'importantes variations liées aux sites adipeux. Ces variations intersites dont les causes ne sont pas encore connues, indiquent cependant que des facteurs circulants ne suffisent pas à expliquer le développement de cette voie métabolique dans l'adipocyte.

Le but de ce travail était de savoir si la capacité du tissu adipeux à estérifier les acides gras pouvait être influencée par la concentration circulante en triglycérides. La suralimentation du nouveau-né par réduction de la taille des portées dès la naissance provoque effectivement une hypertriglycéridémie accompagnée d'hyperinsulinisme. La suralimentation induit rapidement un surdéveloppement du tissu adipeux dû exclusivement d'abord à une hypertrophie adipocytaire. Le modèle permet donc d'étudier les facteurs qui régulent l'activité des enzymes de la voie d'estérification des $A G$ dans l'adipocyte. La suralimentation précoce provoque une augmentation globale de l'activité LPL pendant toute la période qui précède le sevrage. L'hyperphagie induite chez le rat par lésion de l'hypothalamus ventromedian provoque une augmentation de l'activité de cette enzyme (Schnatz et al., 1971). La LPL du tissu adipeux est stimulée après administration d'un régime gras (de Gasquet et Péquignot), 1973). Ainsi, l'activité LPL semble dépendre du niveau des ingesta et l'ensemble de ces résultats suggère que la quantité de triglycérides circulants pourrait être un facteur important de régulation de l'activité de cette enzyme. Toutefois dans tous les modèles cités on observe une hyperinsulinémie qui pourrait rendre compte de l'augmentation de l'activité LPL. En effet le rôle stimulateur de l'insuline sur l'activité LPL est bien connu, in vivo comme in vitro (Hollenberg, 1959 ; Wing et al., 1966) et il est possible que l'augmentation de l'activité LPL chez les rats suralimentés résulte de l'hyperinsulinémie. L'activité LPL exprimée par unité de surface adipocytaire reste plus élevée chez les animaux suralimentés que chez les témoins et ne découle pas de l'augmentation de la taille des adipocytes. Plus vraisemblablement, l'augmentation de l'activité LPL entraîne une hypertrophie adipocytaire chez ces animaux, car la LPL est déjà présente dans les ébauches non différenciées de tissu adipeux de rats nouveau-nés avant toute accumulation notable de triglycérides (Péquignot et al., 1977). La suralimentation précoce n'affecte pas l'activité de l'acyl CoA ligase des rats avant sevrage. Chez le porc, cette enzyme n'est pas non plus modifiée par le régime gras (Steffen et al., 1983). Chez le rat adulte, l'insuline peut stimuler, in vitro, l'activité de l'acyl CoA ligase adipocytaire (Jason et al., 1976). Malgré leur hyperinsulinémie, l'activité de l'acyl CoA ligase reste inchangée chez les animaux suralimentés. II est possible que l'effet de l'insuline in vivo n'apparaisse que tardivement au cours du développement, probablement après le sevrage, et que dans le cas d'une alimentation riche en lipides, l'étape d'activation des AG ne soit pas limitante pour leur stockage. La suralimentation précoce permet le maintien à des niveaux élevés de l'activité GPAT pendant les 10 premiers jours de la vie. Ce fait pourrait être 
dépendant du niveau d'ingesta plutôt que de l'hypertriglycéridémie : en effet l'administration, pendant 4 jours, d'un régime gras (Aas et Daac, 1971) n'a aucune répercussion sur l'activité de cette enzyme, alors qu'une restriction calorique $(1 / 3$ de la ration ad libitum) maintenue pendant 2 semaines, provoque une diminution significative de la synthèse des glycérolipides chez les rats obèses (Jamdar, 1978). L'activité de cette enzyme est fortement corrélée à la taille de l'adipocyte (Jamdar et al., 1981), ce qui explique le niveau plus élevé d'activité chez les animaux suralimentés, à 10 jours, quand ils présentent une hypertrophie adipocytaire.

En conclusion, ces résultats montrent que la suralimentation du nouveau-né provoque rapidement une augmentation de la capacité de stockage des acides gras dans le tissu adipeux; ils suggèrent que la quantité de triglycérides circulants peut réguler dans l'adipocyte, soit directement, soit sous le contrôle de l'insuline, l'activité des enzymes d'estérification des acides gras. La lipoprotéine lipase semble jouer un rôle primordial dans ce phénomène puisque son activité paraît directement corrélée avec le niveau des ingesta.

11 e Réunion du groupe Développement I.N.R.A., Montpellier, 22-24 mai 1985.

Remerciements. - Ce travail a été subventionné par l'Institut National de la Santé et de la Recherche Médicale (CRL $n^{\circ} 82.70 .22$ ).

Les auteurs remercient Mlle $\mathrm{V}$. Resve qui a assuré la dactylographie de ce manuscrit.

\section{Références}

AAS M., DAAC L. N., 1971. Fatty acid activation and acyl transfert in organs from rats in different nutritional state. Biochem. biophys. Acta, 348, 179-188.

COOK J. R., KOZAK L. P., 1982. Sn Glycerol-3-phosphate deshydrogenase gene expression during mouse adipocyte development in vivo. Develop. Biol., 92, 440-448.

CRYER A., JONES H. M., 1978. Changes in lipoprotein lipase activity of white adipose tissue during development of the rat. Biochem. J., 172, 319-325.

FOLCH J., LEES M., SLOANE-STANLEY G. H., 1957. A simple method for isolation and purification of total lipids from animals tissue. J. biol. Chem., 226, 497-509.

GASQUET P. de, PEQUIGNOT E., 1973. Lipoprotein lipase activity in adipose cells from genetically and nutritionally obese rats and mice, p. 47. In J. VAGUE, J. BOYER, Regulation of adipose tissue mass. Excerpta med., Amsterdam.

HAHN P., GREENBERG R., 1968. Incorporation of 1-3 $\mathrm{C}^{14}$ glycerol into triglycerides of adipose tissue from suckling rats. Life Sci., 7, 187-190.

HOLLENBERG C. H., 1959. Effect of nutrition on activity and release of lipase from rat adipose tissue. Am. J. Physiol., 197, 667-670.

JAMDAR S. C., 1978. Glycerolipid biosynthesis in rat adipose tissue. Influence of adipose cell size and site of adipose tissue on triacylglycerol formation in lean and obese rats. Biochem. J., 170, 153-160.

JAMDAR S. C., FALLON H. J., 1973. Glycerolipid synthesis in rat adipose tissue. J. Lipid Res., 14, 509-516.

JAMDAR S. C., OSBORNE L. J., 1981. Glycerolipid biosynthesis in rat adipose tissue. Effect of age, site of adipose tissue and cell size. Biochim. biophys. Acta, 665, 145-153.

JAMDAR S. C., OSBORNE L. J., ZEIGLER J. A., 1981. Glycerolipid biosynthesis in rat adipose tissue. Influence of adipocyte size. Biochem. J., 194, 293-298. 
JASON C. J., POLOKOFF M. A., BELL R. M., 1976. Triacylglycerol synthesis in isolated fat celis : an effect of insulin on microsomal fatty acid coenzyme A ligase activity. J. biol. Chem., 251, $1488-1492$.

KENNEDY G. C., 1957. The effect of age on the somatic and visceral response to overnutrition in the rat. J. Endocrinol., 15, 19-24.

KOZAK L. P., JENSEN J. T., 1974. Genetic and developmental control of multiple forms of glycerol-3-phosphate deshydrogenase. J. biol. Chem., 249, 7775-7781.

LOWRY O. H., ROSEBROUGH N. J., FARR A. L., RANDALL R. J., 1951. Protein measurement with the folin phenol reagent. J. biol. Chem., 193, 265-271.

NILSSON-EHLE P., SCHOTZ M. C., 1976. A stable radioactive substrate emulsion for assay of lipoprotein lipase. J. Lipid Res., 17, 536-541.

PÉQUIGNOT-PLANCHE E., de GASQUET P., BOULANGÉ A., TONNU N. T., 1977. Lipoprotein lipase activity at the onset of development of white adipose tissue in newborn rats. Biochem. J., 162, 461-463.

ROTHBLATT G. H., de MARTINIS F. D., 1977. Release of LPL from rat adipose tissue cells grown in culture. Biochem. Biophys. Res. Commun., 78, 45-50.

SCHNATZ J. D., BERNARDIS L. L., FROHMAN L. A., GOLDMAN J. K., 1971. Hypertriglyceridemia in weaning rats with hypothalamic obesity. Diabetes, 20, 655-663.

STEFFEN D. G., PHINNEY G., BROWN L. J., CHAI E. Y., MERSMANN H. J., 1983. Swine microsomal acyl CoA synthetase activity: effect of age and diet. J. Nutr., 113, 1846-1851.

TANAKA T., HOSAKA K., NUMA S., 1979. Long chain acyl CoA synthetase from rat liver. In LOWENSTEIN J. M., Methods of enzymology, Vol. 71, p. 334. Acad. Press.

WIDDOWSON E. M., MCCANCE R. A., 1960. Some effects of accelerating growth. I. General somatic development. Proc. roy. Soc. London. B, 152, 188-206.

WING D. R., SALAMAN M. R., ROBINSON D. S., 1966. Clearing factor lipase in adipose tissue. Factors affecting the increase in enzyme activity produced in incubation of tissue from starved rats in vitro. Biochem. J., 99, 648.

WISE L. S., GREEN H., 1979. Participation of one isoenzyme of cytosolic glycerophosphate deshydrogenase in the adipose conversion of 3 T3 cells. J. biol. Chem., 254, 373-375. 